\title{
New perspectives on the emergence of $(3+1) D$ expanding space-time in the Lorentzian type IIB matrix model
}

\author{
Jun Nishimura* \\ Theory Center, Institute of Particle and Nuclear Studies, \\ High Energy Accelerator Research Organization (KEK), \\ 1-1 Oho, Tsukuba, Ibaraki 305-0801, Japan \\ Department of Particle and Nuclear Physics, School of High Energy Accelerator Science, \\ Graduate University for Advanced Studies (SOKENDAI), \\ 1-1 Oho, Tsukuba, Ibaraki 305-0801, Japan \\ E-mail: jnishi@post.kek.jp
}

\begin{abstract}
The type IIB matrix model is a promising candidate for a nonperturbative formulation of superstring theory. In the Lorentzian version, in particular, the emergence of $(3+1) D$ expanding spacetime was observed by Monte Carlo studies of this model. Here we provide new perspectives on the $(3+1) D$ expanding space-time that have arised from recent studies. First it was found that the matrix configurations generated by the simulation are singular in that the submatrices representing the expanding 3D space have only two large eigenvalues associated with the Pauli matrices. This problem was conjectured to occur due to the approximation used to avoid the sign problem in simulating the model. In order to confirm this conjecture, the complex Langevin method was applied to overcome the sign problem instead of using the approximation. The results indeed showed a clear departure from the Pauli-matrix structure, while the $(3+1) \mathrm{D}$ expanding behavior remained unaltered. It was also found that classical solutions obtained within a certain ansatz show quite generically a (3+1)D expanding behavior with smooth space-time structure.
\end{abstract}

Corfu Summer Institute 2019 "School and Workshops on Elementary Particle Physics and Gravity" (CORFU2019)

31 August - 25 September 2019

Corfu, Greece

${ }^{*}$ Speaker. 


\section{Introduction}

It is widely accepted that nonperturbative studies are crucial in understanding the dynamics of superstring theory. We consider that this statement is manifested by the emergence of $(3+1) \mathrm{D}$ expanding space-time in the Lorentzian version of the type IIB matrix model [1]. This model was conjectured to be a nonperturbative formulation of superstring theory [2], which is analogous to the lattice gauge theory in QCD. The model has ten bosonic $N \times N$ Hermitian matrices, which are expected to describe ten-dimensional space-time in the large- $N$ limit. The eigenvalue distribution of the ten bosonic matrices can collapse to a lower-dimensional manifold, which may represent the actual space-time dynamically generated in this model. In order for this to happen, the $(9+1) \mathrm{D}$ Lorentz symmetry of the model has to be spontaneously broken.

There are various pieces of evidence for the conjecture that the type IIB matrix model is indeed a nonperturbative formulation of superstring theory. A direct connection to perturbative formulation of superstring theory can be seen by considering the type IIB superstring theory in 10D. First, the action of the model can be regarded as a matrix regularization of the worldsheet action of type IIB superstring theory in the Schild gauge [2]. This does not imply that the matrix model is merely a formulation for the "first quantization" of superstrings because multiple worldsheets appear naturally in the matrix model as block-diagonal configurations, where each block represents the embedding of a single worldsheet into the 10-dimensional target space. Second, under a few reasonable assumptions, the string field Hamiltonian for type IIB superstring theory can be derived from Schwinger-Dyson equations for the Wilson loop operators, which are identified as creation and annihilation operators of strings [3]. This implies that the type IIB matrix model can reproduce perturbative expansions in type IIB superstring theory to all orders.

In these connections to type IIB superstring theory, the target space coordinates are identified with the eigenvalues of the matrices $A_{\mu}$. In particular, this identification is consistent with the supersymmetry algebra of the model, in which the translation that appears from the anti-commutator of supersymmetry generators is identified with the shift symmetry $A_{\mu} \mapsto A_{\mu}+\alpha_{\mu} \mathbf{1}$ of the model, where $\alpha_{\mu} \in \mathbf{R}$. Also the fact that the model has extended $\mathscr{N}=2$ supersymmetry in ten dimensions is consistent with the fact that the model actually includes gravity since it is known in field theory that $\mathscr{N}=1$ supersymmetry is the maximal one that can be achieved in ten dimensions without including gravity.

It was also realized that the five types of superstring theory in ten dimensions are just different descriptions of the same theory. Therefore, it was speculated that the type IIB matrix model actually describes the unique underlying theory, although it takes the form that has explicit connection to perturbative type IIB superstring theory $[2,3]$.

Unfortunately, it is extremely hard to perform Monte Carlo studies of the type IIB matrix model due to the so-called sign problem caused by the complex weight in the partition function. In the Eulicidean version, it comes from the Pfaffian that is obtained by integrating out fermionic matrices, while in the Lorentzian version, it comes from the phase factor $\mathrm{e}^{i S_{\mathrm{b}}}$ with the bosonic action $S_{\mathrm{b}}$. If we treat the phase of the complex weight by reweighting, huge cancellation among configurations with different phases occurs, which makes the calculation impractical. Recently the complex Langevin method (CLM) $[4,5]$ has been attracting much attention as a promising approach to this problem [6-15]. In particular, it was applied successfully to the Euclidean version of the 
6D type IIB matrix model [16], and the spontaneous breaking of the rotational $\mathrm{SO}(6)$ symmetry to $\mathrm{SO}(3)$ suggested by the Gaussian expansion method [17] was confirmed. Recently this work has been extended to the original Euclidean 10D type IIB matrix model [18]. Similarly to the $6 \mathrm{D}$ version, the rotational $\mathrm{SO}(10)$ symmetry was found to be spontaneously broken to $\mathrm{SO}(3)$ as suggested by the Gaussian expansion method [19].

In ref. [1] as well as in more recent work [20-23] on the Lorentzian type IIB matrix model and its simplified models, the sign problem was avoided by integrating out the scale factor of the bosonic matrices by hand, which yields a function of the bosonic action $S_{\mathrm{b}}$ sharply peaked at the origin. Approximating this function by a sharply peaked Gaussian function, we can perform Monte Carlo simulations without the sign problem. The emergence of $(3+1) \mathrm{D}$ expanding spacetime was obtained in this way [1]. The expanding behavior for a longer time was investigated by simulating the simplified models. The obtained results suggested a scenario for the full model that the expansion is exponential at early times [20], which is reminiscent of the inflation, and that it turns into a power law [21] at later times, which is reminiscent of the Friedmann-Robertson-Walker universe in the radiation dominated era. See also refs. [24-26] for closely related work.

It has been found recently, however, that the matrix configurations generated by the simulation is singular in that the submatrices representing the expanding 3D space have only two large eigenvalues associated with the Pauli matrices [27]. This problem has been attributed to the aforementioned approximation used to avoid the sign problem since the function obtained after integrating out the scale factor is actually complex-valued, and the effect of the phase is not taken into account. It was realized that the approximation actually amounts to replacing the phase factor $\mathrm{e}^{i S_{\mathrm{b}}}$ by a positive definite weight $\mathrm{e}^{c S_{\mathrm{b}}}$ with some constant $c>0$. This new interpretation of the simulation provides clear understanding of the observed Pauli-matrix structure and the (3+1)D expanding behavior. It has also been argued that a regular space-time may be obtained if the phase factor $\mathrm{e}^{i S_{\mathrm{b}}}$ is used correctly. This is a very nontrivial issue, however, since losing the Pauli-matrix structure may also imply losing the $(3+1) \mathrm{D}$ expanding behavior at the same time. A piece of evidence that this is possible has been provided by generating classical solutions of the Lorentzian type IIB matrix model [28], where it was found that the solutions obtained within a certain ansatz show quite generically a (3+1)D expanding behavior with smooth space-time structure.

In ref. [29], which we review in this article, we addressed this issue by using the CLM to solve the sign problem instead of using the aforementioned approximation. Note that the Lorentzian type IIB matrix model needs to be regularized in some way or another because the phase factor $\mathrm{e}^{i S_{\mathrm{b}}}$ in the partition function cannot suppress the contribution from the bosonic matrices with arbitrary large elements. ${ }^{1}$ Here we use the infrared cutoffs on both the spatial and temporal matrices analogous to the ones used in the previous work [1]. We also find it useful to introduce two deformation parameters $(s, k)$, which correspond to the Wick rotations on the worldsheet and in the target space, respectively. These parameters enable us to interpolate between the Lorentzian version $(s, k)=$ $(0,0)$ and the Euclidean version $(s, k)=(1,1)$.

First we focus on $(s, k)=(-1,0)$ in the deformation parameter space, where we do not have the sign problem. In fact, this case corresponds to the approximate model investigated in our

\footnotetext{
${ }^{1}$ This situation is in sharp contrast to the Euclidean version [30,31], in which the phase factor $\mathrm{e}^{i S_{\mathrm{b}}}$ is replaced by $\mathrm{e}^{-S_{\mathrm{b}}^{(\mathrm{E})}}$, where $S_{\mathrm{b}}^{(\mathrm{E})}$ is a real non-negative quantity.
} 
previous work. We observe the emergence of $(3+1) \mathrm{D}$ expanding space-time with the Pauli-matrix structure. Then we tune the worldsheet deformation parameter $s$ close to that for the Lorentzian model $(s=0)$ keeping the target space deformation parameter $k$ in such a way that the space-time noncommutativity is minimized. There, we find it possible to obtain smoother space-time structure without losing the $(3+1) \mathrm{D}$ expanding behavior. The deviation from the Pauli-matrix structure was not seen for the matrix size $N \leq 64$ within the parameter region that can be explored by the CLM, and it becomes more prominent as we increase $N$ from 128 to 192 . We consider that the two deformation parameters $s$ and $k$ should be tuned eventually to $(s, k)=(0,0)$ in the large- $N$ limit. Whether a smooth classical space-time picture appears in that limit at sufficiently late time is an important open question, which can be answered along the line of this research.

The rest of this article is organized as follows. In section 2 we define the Lorentzian type IIB matrix model and introduce the infrared cutoffs as well as the two deformation parameters $s$ and $k$. In section 3 we discuss how we apply the CLM to the Lorentzian type IIB matrix model. In section 4 we focus on $(s, k)=(-1,0)$ in the deformation parameter space, which corresponds to the approximate model investigated in the previous work. Indeed we observe the emergence of $(3+1) \mathrm{D}$ expanding space-time with the Pauli-matrix structure. In section 5] we show our results for the worldsheet deformation parameter $s$ close to that for the Lorentzian model $(s=0)$ with the target space deformation parameter $k$ chosen in such a way that the space-time noncommutativity is minimized. We observe a clear departure from the Pauli-matrix structure, while the $(3+1) \mathrm{D}$ expanding behavior is still being observed. Section 6 is devoted to a summary and discussions.

\section{Definition of the Lorentzian type IIB matrix model}

The action of the Lorentzian type IIB matrix model is given by [2]

$$
\begin{aligned}
S & =S_{\mathrm{b}}+S_{\mathrm{f}}, \\
S_{\mathrm{b}} & =\frac{1}{4 g^{2}} \operatorname{Tr}\left(\left[A_{\mu}, A_{v}\right]\left[A^{\mu}, A^{v}\right]\right), \\
S_{\mathrm{f}} & =-\frac{1}{2 g^{2}} \operatorname{Tr}\left(\Psi_{\alpha}\left(\mathscr{C} \Gamma^{\mu}\right)_{\alpha \beta}\left[A_{\mu}, \Psi_{\beta}\right]\right),
\end{aligned}
$$

where the bosonic variables $A_{\mu}(\mu=0, \ldots, 9)$ and the fermionic variables $\Psi_{\alpha}(\alpha=1, \ldots, 16)$ are $N \times N$ Hermitian matrices. $\Gamma^{\mu}$ are 10D gamma-matrices after the Weyl projection and $\mathscr{C}$ is the charge conjugation matrix. The "coupling constant" $g$ is merely a scale parameter in this model since it can be absorbed by rescaling $A_{\mu}$ and $\Psi$ appropriately. The indices $\mu$ and $\nu$ are contracted using the Lorentzian metric $\eta_{\mu \nu}=\operatorname{diag}(-1,1, \ldots, 1)$. The Euclidean version can be obtained by making a "Wick rotation" $A_{0}=i A_{10}$, where $A_{10}$ is Hermitian.

The partition function for the Lorentzian version is proposed in ref. [1] as

$$
Z=\int d A d \Psi e^{i S}
$$

with the action (2.1). The " $i$ " in front of the action is motivated from the fact that the string worldsheet metric should also have a Lorentzian signature. By integrating out the fermionic matrices, we obtain the Pfaffian

$$
\int d \Psi e^{i S_{\mathrm{f}}}=\operatorname{Pf} \mathscr{M}(A)
$$


which is real unlike in the Euclidean case [32]. Note also that the bosonic action (2.2) can be written as

$$
S_{\mathrm{b}}=\frac{1}{4 g^{2}} \operatorname{Tr}\left(F_{\mu v} F^{\mu v}\right)=\frac{1}{4 g^{2}}\left\{-2 \operatorname{Tr}\left(F_{0 i}\right)^{2}+\operatorname{Tr}\left(F_{i j}\right)^{2}\right\}
$$

where we have introduced the Hermitian matrices $F_{\mu \nu}=i\left[A_{\mu}, A_{v}\right]$. Since the two terms in the last expression have opposite signs, $S_{\mathrm{b}}$ is not positive semi-definite, and it is not bounded from below.

In order to make the partition function (2.4) finite, we need to introduce infrared cutoffs in both the temporal and spatial directions, for instance, as

$$
\begin{aligned}
& \frac{1}{N} \operatorname{Tr}\left(A_{0}\right)^{2} \leq \kappa L^{2}, \\
& \frac{1}{N} \operatorname{Tr}\left(A_{i}\right)^{2} \leq L^{2} .
\end{aligned}
$$

We can use the $\mathrm{SU}(N)$ symmetry of the model to bring the temporal matrix $A_{0}$ into the diagonal form

$$
A_{0}=\operatorname{diag}\left(\alpha_{1}, \ldots, \alpha_{N}\right), \quad \text { where } \alpha_{1}<\cdots<\alpha_{N} .
$$

By "fixing the gauge" in this way, we can rewrite the partition function 2.4 as

$$
\begin{aligned}
Z & =\int \prod_{a=1}^{N} d \alpha_{a} \Delta(\alpha)^{2} \int d A_{i} e^{i S_{\mathrm{b}}} \operatorname{Pf} \mathscr{M}(A), \\
\Delta(\alpha) & \equiv \prod_{a>b}^{N}\left(\alpha_{a}-\alpha_{b}\right)
\end{aligned}
$$

where $\Delta(\alpha)$ is the van der Monde determinant. The factor $\Delta(\alpha)^{2}$ in 2.10 appears from the FadeevPopov procedure for the gauge fixing, and it acts as a repulsive potential between the eigenvalues $\alpha_{i}$ of $A_{0}$.

We can extract a time-evolution from configurations generated by simulating (2.10). A crucial observation is that the spatial matrices $A_{i}$ have a band-diagonal structure in the $\mathrm{SU}(N)$ basis in which $A_{0}$ has the diagonal form 2.9]. More precisely, there exists some integer $n$ such that the elements of spatial matrices $\left(A_{i}\right)_{a b}$ for $|a-b|>n$ are much smaller than those for $|a-b| \leq n$. Based on this observation, we may naturally consider $n \times n$ submatrices of $A_{i}$ defined as

$$
\left(\bar{A}_{i}\right)_{I J}(t) \equiv\left(A_{i}\right)_{v+I, v+J}
$$

where $I, J=1, \ldots, n, v=0,1, \ldots, N-n$, and $t$ is defined by

$$
t=\frac{1}{n} \sum_{I=1}^{n} \alpha_{v+I}
$$

We interpret the $\bar{A}_{i}(t)$ as representing the state of the universe at time $t$.

Using $\bar{A}_{i}(t)$, we can define, for example, the extent of space at time $t$ as

$$
R^{2}(t)=\left\langle\frac{1}{n} \operatorname{tr} \sum_{i}\left(\bar{A}_{i}(t)\right)^{2}\right\rangle,
$$


where the symbol tr represents a trace over the $n \times n$ submatrix. We also define the "moment of inertia tensor"

$$
T_{i j}(t)=\frac{1}{n} \operatorname{tr}\left(\bar{A}_{i}(t) \bar{A}_{j}(t)\right),
$$

which is a $9 \times 9$ real symmetric matrix. The eigenvalues of $T_{i j}(t)$, which we denote by $\lambda_{i}(t)$ with the order

$$
\lambda_{1}(t)>\lambda_{2}(t)>\cdots>\lambda_{9}(t)
$$

represent the spatial extent in each of the nine directions at time $t$. Note that the expectation values $\left\langle\lambda_{i}(t)\right\rangle$ tend to be equal in the large- $N$ limit if the $\mathrm{SO}(9)$ symmetry is not spontaneously broken. This is the case at early times of the time-evolution. After a critical time $t_{\mathrm{c}}$, on the other hand, it was found [1] that the three largest eigenvalues $\left\langle\lambda_{i}(t)\right\rangle(i=1,2,3)$ become significantly larger than the rest, which implies that the $\mathrm{SO}(9)$ symmetry is spontaneously broken down to $\mathrm{SO}(3)$.

Here we introduce two deformation parameters $s$ and $k$, which correspond to Wick rotations on the worldsheet and in the target space, respectively. Let us introduce $\tilde{S}=-i S_{\mathrm{b}}$ so that the factor $e^{i S_{\mathrm{b}}}$ in the partition function (2.10) is rewritten as $e^{-\tilde{S}}$. We introduce the first parameter $s(-1 \leq s \leq 1)$ corresponding to the Wick rotation on the worldsheet as

$$
\tilde{S}=-i N \beta \mathrm{e}^{i s \pi / 2}\left\{-\frac{1}{2} \operatorname{Tr}\left(F_{0 i}\right)^{2}+\frac{1}{4} \operatorname{Tr}\left(F_{i j}\right)^{2}\right\}
$$

where $\beta=\frac{1}{g^{2} N}$. The second parameter $k(0 \leq k \leq 1)$ corresponding to the Wick rotation in the target space can be introduced by the replacement $A_{0} \mapsto \mathrm{e}^{-i k \pi / 2} A_{0}$. The action 2.17 becomes

$$
\tilde{S}=-i N \beta \mathrm{e}^{i s \pi / 2}\left\{-\frac{1}{2} \mathrm{e}^{-i k \pi} \operatorname{Tr}\left(F_{0 i}\right)^{2}+\frac{1}{4} \operatorname{Tr}\left(F_{i j}\right)^{2}\right\},
$$

and the $\operatorname{Pf} \mathscr{M}(A)$ in 2.10 should be replaced by Pf $\mathscr{M}\left(\mathrm{e}^{-i k \pi / 2} A_{0}, A_{i}\right)$. The Lorentzian model is retrieved at $(s, k)=(0,0)$, whereas the Euclidean model corresponds to setting $(s, k)=(1,1)$.

Note that the coefficient of the first term in (2.18) can be made real non-negative by choosing the parameters so that $i \mathrm{e}^{i s \pi / 2} \mathrm{e}^{-i k \pi}=1$, which implies $k=(1+s) / 2$. For this choice, the bosonic action is most effective in minimizing the noncommutativity between the spatial matrices $A_{i}$ and the temporal matrix $A_{0}$. For $0 \leq k<s / 2$, on the other hand, the real part of the coefficient becomes negative, which favors maximum noncommutativity between $A_{i}$ and $A_{0}$. As a result, the eigenvalues of $A_{0}$ lump up into two clusters separated from each other, and we cannot obtain a continuous time. The Lorentzian model $(s, k)=(0,0)$ lies on the boundary of this unphysical region. Here we keep away from this region by restricting ourselves to the cases satisfying $k=(1+s) / 2$.

Taking into account the infrared cutoffs (2.7) and (2.8), we arrive at the partition function

$$
\begin{aligned}
Z= & \int \prod_{a=1}^{N} d \alpha_{a} \Delta(\alpha)^{2} \int d A_{i} e^{-\tilde{S}} \operatorname{Pf} \mathscr{M}\left(\mathrm{e}^{-i k \pi / 2} A_{0}, A_{i}\right) \times \\
& \theta\left(\kappa L^{2}-\frac{1}{N} \operatorname{Tr}\left(A_{0}\right)^{2}\right) \theta\left(L^{2}-\frac{1}{N} \operatorname{Tr}\left(A_{i}\right)^{2}\right),
\end{aligned}
$$

where $\theta(x)$ is the Heaviside step function and $\tilde{S}$ is given by (2.18). By rescaling $A_{\mu} \mapsto L A_{\mu}$ and $\beta \mapsto L^{-4} A_{\mu}$, we can set $L=1$ without loss of generality. 


\section{The complex Langevin method}

In this section, we review the CLM and discuss how we apply it to the Lorentzian type IIB matrix model.

\subsection{Brief review of the CLM}

Let us consider a system

$$
Z=\int d x w(x)
$$

of $N$ real variables $x_{k}(k=1, \cdots, N)$ as a simple example. Here the weight $w(x)$ is a complex-valued function, which causes the sign problem.

In the CLM, the original real variables $x_{k}$ are complexified as $x_{k} \rightarrow z_{k}=x_{k}+i y_{k} \in \mathbb{C}$ and one considers a fictitious time evolution of the complexified variables $z_{k}$ using the complex Langevin equation given, in its discretized form, by

$$
z_{k}^{(\eta)}(t+\varepsilon)=z_{k}^{(\eta)}(t)+\varepsilon v_{k}\left(z^{(\eta)}(t)\right)+\sqrt{\varepsilon} \eta_{k}(t),
$$

where $t$ is the fictitious time with a stepsize $\varepsilon$. The second term $v_{k}(z)$ on the right-hand side is called the drift term, which is defined by holomorphic extension of the one

$$
v_{k}(x)=w(x)^{-1} \frac{\partial w(x)}{\partial x_{k}}
$$

for the real variables $x_{k}$. The variables $\eta_{k}(t)$ appearing on the right-hand side of eq. (3.2) are a real Gaussian noise with the probability distribution $\propto e^{-\frac{1}{4} \Sigma_{t} \eta_{k}(t)^{2}}$, which makes the time-evolved variables $z_{k}^{(\eta)}(t)$ stochastic. The expectation values with respect to the noise $\eta_{k}(t)$ are denoted as $\langle\cdots\rangle_{\eta}$ in what follows.

Let us consider the expectation value of an observable $\mathscr{O}(x)$. In the CLM, one computes the expectation value of the holomorphically extended observable $\mathscr{O}(x+i y)$ as

$$
\left\langle\mathscr{O}\left(z^{(\eta)}(t)\right)\right\rangle_{\eta}=\int d x d y \mathscr{O}(x+i y) P(x, y ; t),
$$

where $P(x, y ; t)$ is the probability distribution of $x^{(\eta)}(t)$ and $y^{(\eta)}(t)$ defined by

$$
P(x, y ; t)=\left\langle\delta\left(x-x^{(\eta)}(t)\right) \delta\left(y-y^{(\eta)}(t)\right)\right\rangle_{\eta} .
$$

Then, the correct convergence of the CLM implies the equality

$$
\lim _{t \rightarrow \infty} \lim _{\varepsilon \rightarrow 0}\left\langle\mathscr{O}\left(z^{(\eta)}(t)\right)\right\rangle_{\eta}=\frac{1}{Z} \int d x \mathscr{O}(x) w(x),
$$

where the right-hand side is the expectation value of $\mathscr{O}(x)$ in the original theory (3.1). A proof of eq. (3.6) was given in refs. $[7,8]$, where the notion of the time-evolved observable $\mathscr{O}(z ; t)$ plays a crucial role. In particular, it was pointed out that the integration by parts used in the argument cannot be justified when the probability distribution (3.5) falls off slowly in the imaginary direction. In ref. [10], it was noticed that the wrong convergence associated with the zeroes of the fermion 
determinant [33] is actually due to the slow fall-off of the probability distribution (3.5) toward the singularities of the drift term.

While this argument provided theoretical understanding of the cases in which the CLM gives wrong results, the precise condition on the probability distribution was not specified. Furthermore, there is actually a subtlety in defining the time-evolved observable. Recently ref. [12] provided a refined argument for justification of the CLM [12], which showed that the probability for the drift term $v_{k}(z)$ to become large has to be suppressed strongly enough. More precisely the histogram of the magnitude of the drift term should fall off exponentially or faster. This criterion tells us whether the results obtained by the CLM are reliable or not. Its usefulness was demonstrated in ref. [12] for two simple one-variable models and also for systems with many degrees of freedom [34].

\subsection{Applying the CLM to the Lorentzian model}

Let us apply the CLM to the model (2.19). From now on, we omit the Pfaffian and consider the $6 \mathrm{D}$ version, which consists of $A_{0}$ and $A_{i}(i=1, \cdots, 5)$, for simplicity.

The first step of the CLM is to complexify the real variables. As for the spatial matrices $A_{i}$, we simply treat them as general complex matrices instead of Hermitian matrices. As for the temporal matrix $A_{0}$, which is diagonalized as 2.9 , we have to take into account the ordering of the eigenvalues. For that purpose, we make the change of variables as

$$
\alpha_{1}=0, \quad \alpha_{2}=\mathrm{e}^{\tau_{1}}, \quad \alpha_{3}=\mathrm{e}^{\tau_{1}}+\mathrm{e}^{\tau_{2}}, \quad \ldots, \quad \alpha_{N}=\sum_{a=1}^{N-1} \mathrm{e}^{\tau_{a}}
$$

so that the ordering is implemented automatically, and then complexify $\tau_{a}(a=1, \cdots, N-1)$. We have chosen to set $\alpha_{1}=0$ using the shift symmetry $A_{0} \mapsto A_{0}+$ const. 1 of the action. In order to respect this symmetry, we impose the cutoff like 2.7] only on the traceless part $\tilde{A}_{0}=A_{0}-\frac{1}{N} \operatorname{Tr} A_{0}$ in what follows.

The Heaviside function in 2.19 is difficult to treat in the CLM as it is. Here we mimic its effect by introducing the potential

$$
S_{\text {pot }}=\frac{1}{p} \gamma_{\mathrm{s}}\left(\frac{1}{N} \operatorname{Tr}\left(A_{i}\right)^{2}-1\right)^{p}+\frac{1}{p} \gamma_{\mathrm{t}}\left(\frac{1}{N} \operatorname{Tr}\left(\tilde{A}_{0}\right)^{2}-\kappa\right)^{p},
$$

where the power $p$ is set to $p=4$, and the coefficients $\gamma_{\mathrm{s}}$ and $\gamma_{\mathrm{t}}$ are chosen to be large enough to make $\frac{1}{N} \operatorname{Tr}\left(A_{i}\right)^{2}$ and $\frac{1}{N} \operatorname{Tr}\left(\tilde{A}_{0}\right)^{2}$ fluctuate around some constants. ${ }^{2}$ The effective action then reads

$$
\begin{aligned}
S_{\mathrm{eff}} & =N \beta e^{-i \frac{\pi}{2}(1-s)}\left\{\frac{1}{2} e^{-i k \pi} \operatorname{Tr}\left[A_{0}, A_{i}\right]^{2}-\frac{1}{4} \operatorname{Tr}\left[A_{i}, A_{j}\right]^{2}\right\} \\
& +\frac{1}{p} \gamma_{\mathrm{s}}\left(\frac{1}{N} \operatorname{Tr}\left(A_{i}\right)^{2}-1\right)^{p}+\frac{1}{p} \gamma_{\mathrm{t}}\left(\frac{1}{N} \operatorname{Tr}\left(\tilde{A}_{0}\right)^{2}-\kappa\right)^{p} \\
& -\log \Delta(\alpha)-\sum_{a=1}^{N-1} \tau_{a},
\end{aligned}
$$

\footnotetext{
${ }^{2}$ This appears different from imposing the inequalities 2.7] and 2.8), but the difference is not important since the inequalities are typically saturated due to entropic effects.
} 
where the last term comes from the Jacobian associated with the change of variables 3.7). The complex Langevin equation is given by

$$
\begin{aligned}
\frac{d \tau_{a}}{d t} & =-\frac{\partial S_{\mathrm{eff}}}{\partial \tau_{a}}+\eta_{a}(t), \\
\frac{d\left(A_{i}\right)_{a b}}{d t} & =-\frac{\partial S_{\mathrm{eff}}}{\partial\left(A_{i}\right)_{b a}}+\left(\eta_{i}\right)_{a b}(t),
\end{aligned}
$$

where the $\eta_{a}(t)$ in the first equation are random real numbers obeying the probability distribution $\exp \left(-\frac{1}{4} \int d t \sum_{a}\left\{\eta_{a}(t)\right\}^{2}\right)$ and the $\eta_{i}(t)$ in the second equation are random Hermitian matrices obeying the probability distributions $\exp \left(-\frac{1}{4} \int d t \sum_{i} \operatorname{Tr}\left\{\eta_{i}(t)\right\}^{2}\right)$.

The expectation values of observables can be calculated by defining them holomorphically for complexified $\tau_{a}$ and $A_{i}$ and taking an average using the configurations generated by solving the discretized version of (3.10) for sufficiently long time. In order for this method to work, the probability distribution of the drift terms, namely the first terms on the right-hand side of (3.10), has to fall off exponentially [12]. We have checked that this criterion is indeed satisfied for all the values of parameters used in this paper.

\section{Emergence of (3+1)D expanding behavior}

In this section we consider $(s, k)=(-1,0)$ in the parameter space. The action is given by

$$
S=N \beta\left\{-\frac{1}{2} \operatorname{Tr}\left[A_{0}, A_{i}\right]^{2}+\frac{1}{4} \operatorname{Tr}\left[A_{i}, A_{j}\right]^{2}\right\},
$$

which is real, and the CLM reduces to the ordinary Langevin method. The first term in 4.1) tries to minimize the space-time noncommutativity, which has the effects of making the spatial matrices close to diagonal in the basis 2.9. . On the other hand, the second term favors maximal noncommutativity among spatial matrices.

Figure 1 shows our results $^{3}$ for $N=128, \kappa=0.02, \beta=8$. The block size for (2.12) is chosen to be $n=16$. In the Left panel, we plot the eigenvalues $\lambda_{i}(t)$ of $T_{i j}(t)$, which shows that only three out of five eigenvalues become large in the time region around the peak. This suggests that the rotational $\mathrm{SO}(5)$ symmetry of the $6 \mathrm{D}$ bosonic model is broken down to $\mathrm{SO}(3)$ in that time region. These results are qualitatively the same as what has been obtained in ref. [21], which is consistent with the speculation [27] that the previous simulations correspond to the parameter choice $(s, k)=(-1,0)$.

As is known from the previous work [1], the time difference between the peak and the critical time at which the SSB occurs increases in physical units as we take the large- $N$ limit. Therefore, the reflection symmetry with respect to $t$ does not necessarily imply that the Big Crunch occurs in the finite future.

The mechanism of this SSB can be understood as follows [27]. Since the first term in 44.1) favors $A_{i}$ close to diagonal, we may consider the submatrices $\bar{A}_{i}(t)$ as the effective degrees of freedom. The infrared cutoff (2.8) fixes $\operatorname{Tr}\left\{\bar{A}_{i}(t)\right\}^{2}$ to some constant, and the second term in (4.1) favors maximal noncommutativity between $\bar{A}_{i}(t)$. According to the argument in ref. [1], this leads

\footnotetext{
${ }^{3}$ Here and hence forth, we plot the results obtained for one thermalized configuration.
} 

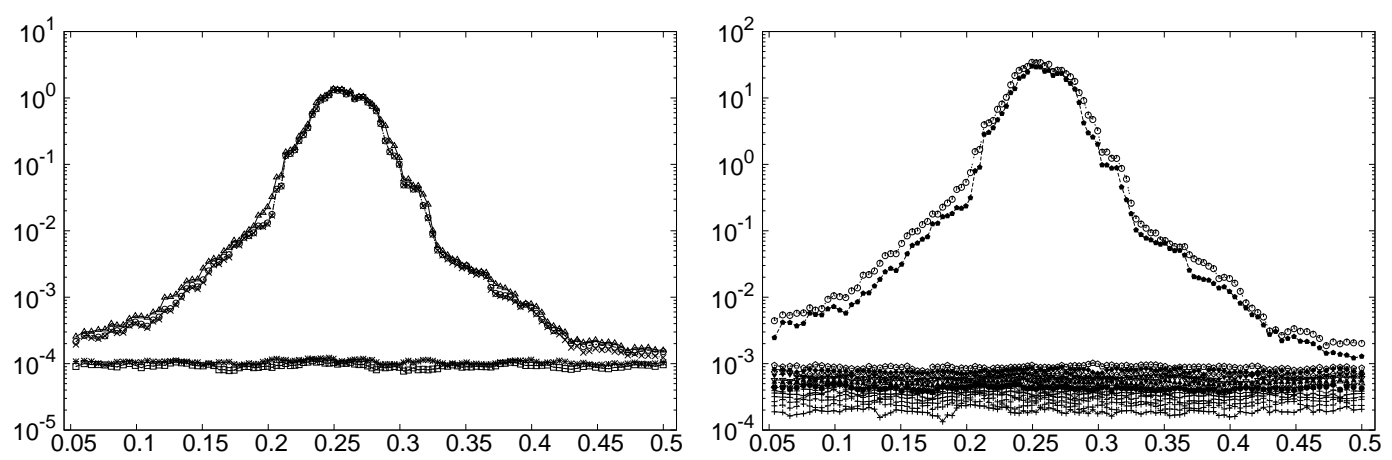

Figure 1: Results for $(s, k)=(-1,0), N=128, \kappa=0.02, \beta=8, n=16$ are shown. (Left) The five eigenvalues of the moment of inertia tensor are plotted against $t$ in the log scale. (Right) The 16 eigenvalues of the matrix $Q(t)$ are plotted against $t$ in the log scale.

to $\bar{A}_{i}(t) \propto \sigma_{i} \oplus \mathbf{0}_{n-2}$ for $i=1,2,3$ and $\bar{A}_{i}(t)=\mathbf{0}_{n}$ for $i \geq 4$ up to $\mathrm{SO}(5)$ rotations, where $\sigma_{i}$ are the Pauli matrices. In order to confirm this mechanism, we calculate the matrix

$$
Q(t)=\sum_{i=1}^{5}\left\{\bar{A}_{i}(t)\right\}^{2},
$$

and plot the eigenvalues of $Q(t)$ in Fig. 1 (Right). Indeed we find that only two of them are large, while the rest are very small in the time region in which the SSB occurs.

\section{Emergence of a smooth space-time}

In this section we tune the worldsheet deformation parameter $s$ to some values near $s=0$, which is the target value for the Lorentzian model, keeping the target-space deformation parameter $k$ to be $k=(1+s) / 2$, which minimizes the space-time noncommutativity. The action reads

$$
S=N \beta\left\{-\frac{1}{2} \operatorname{Tr}\left[A_{0}, A_{i}\right]^{2}-\mathrm{e}^{-i \frac{\pi}{2}(1-s)} \frac{1}{4} \operatorname{Tr}\left[A_{i}, A_{j}\right]^{2}\right\} .
$$

The only difference from 4.1 is the second term with the coefficient $\mathrm{e}^{-i \frac{\pi}{2}(1-s)}$ whose real part changes its sign at $s=0$. This implies, in particular, that for $s>0$ the second term starts to minimize the noncommutativity among the spatial matrices. Therefore, we may anticipate a drastic change of the behavior around $s=0$. In fact, for the values of $s$ below what is reported below, we do not see any qualitative difference from the results obtained at $s=-1$.

Figure 2 2 shows our results for $N=128, \kappa=0.02, \beta=8, n=16$ with $(s, k)=(-0.004,0.498)$. Unlike the $(s, k)=(-1,0)$ case, the action becomes complex for $s>-1$ in general. Therefore, the quantity such as $R^{2}(t)$ defined in 2.14 is not guaranteed to be real positive. In the Top panel, we plot the real and imaginary parts of $R^{2}(t)$. We find that $R^{2}(t)$ is dominated by the real part near the peak.

Let us also take a look at the "Hermiticity norm" for $\bar{A}_{i}(t)$ defined by

$$
h(t)=\frac{-\operatorname{Tr}\left(\bar{A}_{i}(t)-\bar{A}_{i}(t)^{\dagger}\right)^{2}}{4 \operatorname{Tr}\left(\bar{A}_{i}(t)^{\dagger} \bar{A}_{i}(t)\right)},
$$



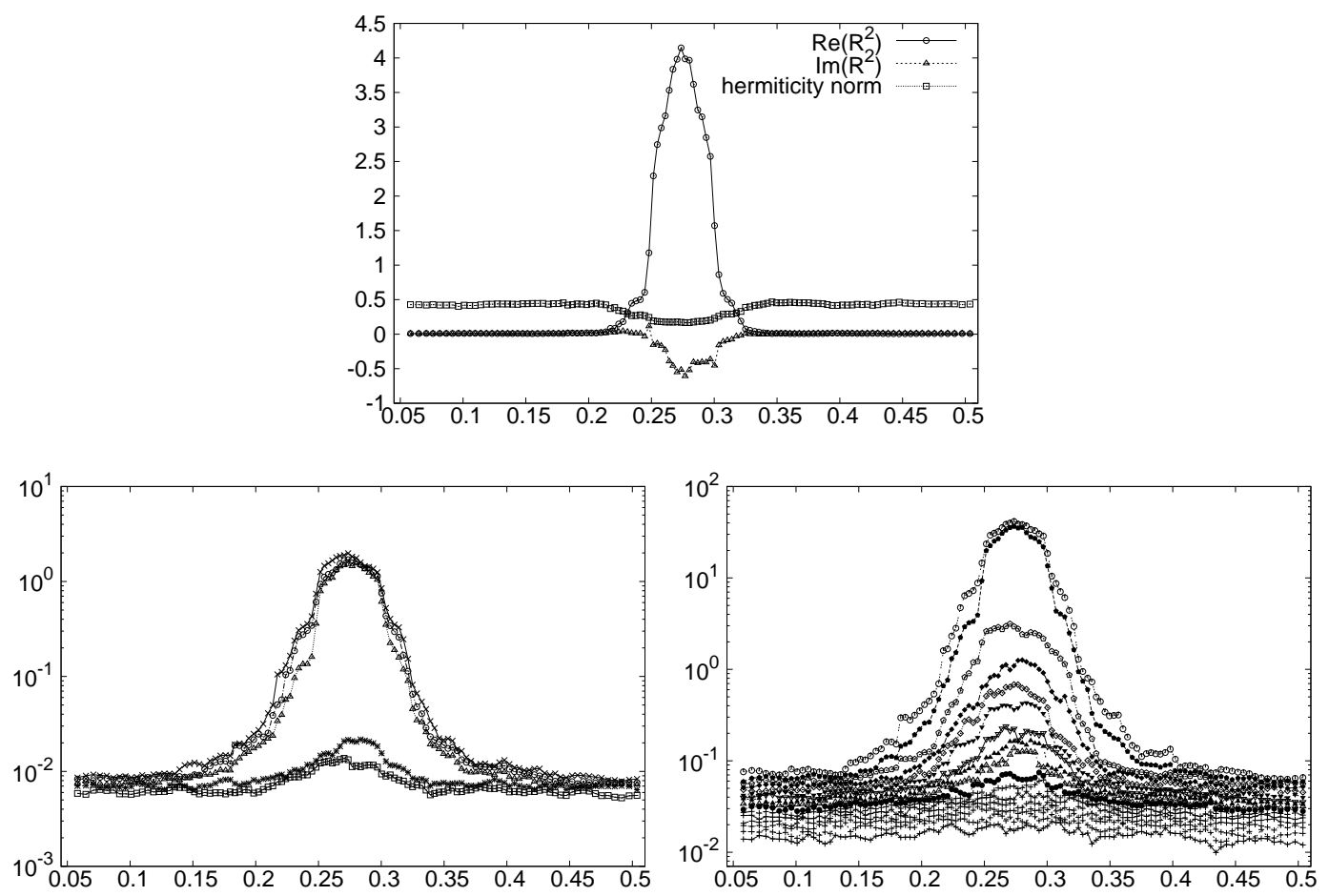

Figure 2: Results for $(s, k)=(-0.004,0.498), N=128, \kappa=0.02, \beta=8, n=16$ are shown. (Top) The real and imaginary parts of $R^{2}(t)$ are plotted against $t$. The Hermiticity norm $h(t)$ of the matrix $\bar{A}_{i}(t)$ is also plotted. (Bottom-Left) The five eigenvalues of the moment of inertia tensor are plotted against $t$ in the log scale. (Bottom-Right) The 16 eigenvalues of the matrix $Q(t)$ are plotted against $t$ in the log scale.

using a configuration generated by the simulation. The result is plotted in the Top panel as well. Note that $h(t)=0$ implies that the matrices $\bar{A}_{i}(t)$ are all Hermitian, while $h(t)=1$ implies that they are all anti-Hermitian. We find that $h(t)$ is small and hence the $\bar{A}_{i}(t)$ are close to Hermitian near the peak, which is consistent with our observation that $R^{2}(t)$ is dominated by the real part in this region. This property supports our previous speculation $[35,36]$ that some classical solution, which is typically represented by a real configuration, dominates the path integral in the time region near the peak due to the expansion of space.

In the Bottom panels, we plot the same quantities ${ }^{4}$ as in Fig. 1. From the left panel, we observe that $(3+1) \mathrm{D}$ expanding behavior persists at $s \sim 0$, while the right panel reveals a clear departure from the Pauli-matrix structure.

We have also investigated the model with $N<128$. For $N=32,64$, while the results at $(s, k)=(-1,0)$ are similar to those for $N=128$, the departure from the Pauli-matrix structure does not show up at all even at $s \sim 0$. As we increase $s$ further with $k=(1+s) / 2$, the Hermiticity of the configurations is completely lost, and the criterion for justifying the CLM is found to be violated.

${ }^{4}$ In fact, it is not straightforward to calculate the expectation values of the eigenvalues of $T_{i j}(t)$ and $Q(t)$ in the CLM respecting holomorphicity because of their multi-valuedness. Here we simply evaluate $T_{i j}(t)$ and $Q(t)$ using the Hermitian part of $\bar{A}_{i}(t)$ from one configuration generated by the complex Langevin simulation, and plot their eigenvalues. 
Thus the use of large values of $N$ seems to be crucial in investigating the model near the target values $(s, k)=(0,0)$.

\section{Summary and discussions}

We consider that by now there is surmounting evidence that the Lorentzian type IIB matrix model is a promising candidate for a nonperturbative formulation of superstring theory. Unfortunately Monte Carlo studies of this model are extremely hard due to the sign problem caused by the phase factor $\mathrm{e}^{i S_{\mathrm{b}}}$ in the partition function. Previous work avoided this problem by integrating out the scale factor of the bosonic matrices and using an approximation. However, it was noticed recently that this approximation actually amounts to replacing $\mathrm{e}^{i S_{\mathrm{b}}}$ by $\mathrm{e}^{c S_{\mathrm{b}}}$ for some $c>0$. This suggests the importance of studying the model without such an approximation.

In this article we have reviewed the results of ref. [29], where we investigated the space-time structure based on the complex Langevin simulation of the $(5+1) \mathrm{D}$ bosonic version of the model with the deformation parameters $s$ and $k$ corresponding to the Wick rotations on the worldsheet and in the target space, respectively, The original model corresponds to $(s, k)=(0,0)$, whereas our previous simulations were speculated to correspond to the $(s, k)=(-1,0)$ case [27]. Our results for $(s, k)=(-1,0)$ indeed reproduced the $(3+1)$ D expanding behavior with the Pauli-matrix structure as expected. Then we tuned the parameter $s$ towards the region $s \sim 0$ restricting ourselves to $k=(1+s) / 2$ in order to stabilize our simulation. The results indeed showed a clear departure from the Pauli-matrix structure, while the $(3+1) \mathrm{D}$ expanding behavior is kept intact. The spatial matrices turn out to be close to Hermitian near the peak of the spatial extent $R^{2}(t)$ even for $s \sim 0$, which confirms our expectation $[35,36]$ that some classical solution dominates at late times.

The appearance of the Pauli-matrix structure for $(s, k)=(-1,0)$ is due to the $\operatorname{Tr}\left(F_{i j}\right)^{2}$ term in the action, which tries to make the spatial matrices $A_{i}$ maximally noncommutative. The situation changes drastically around $s=0$, where the coefficient of the $\operatorname{Tr}\left(F_{i j}\right)^{2}$ term becomes pure imaginary. On the other hand, there are infinitely many classical solutions [28,35-37], which have (3+1)D expanding behavior without the Pauli-matrix structure. (See also refs. [38-43] for related work.) We therefore consider it possible that the space-time structure becomes smooth without losing the $(3+1) \mathrm{D}$ expanding behavior in the large- $N$ limit. The departure from the Pauli-matrix structure observed at $s \sim 0$ supports this possibility.

As future prospects, the most important thing to do is to repeat the same analysis with increased matrix size $N$. In particular, we need to confirm the appearance of a smooth space-time at $(s, k) \sim(0,0)$. While this issue may not depend much on the effects of the fermionic matrices, it would be certainly desirable to include them eventually. Unfortunately, this is not straightforward since the complex Langevin method may suffer from the singular-drift problem due to the near-zero eigenvalues of the Dirac operator. The deformation technique [13] used successfully in studying the Euclidean version $[16,18]$ is worth trying, though. We consider that the dominance of classical solutions at late times $[35,36]$ supported by our results is important because it enables us to understand possible late-time behaviors of this model by solving classical equations of motion. For instance, we may try to find classical solutions [44-47], which can accommodate Standard Model particles as excitations around them. See ref. [28] for a recent work in this direction, where we also find that smooth space-time structure is obtained quite generically in classical solutions. 
The new perspectives on the (3+1)D expanding space-time in the Lorentzian type IIB matrix model that have arised recently make this model more promising as a nonperturbative formulation of superstring theory. In particular, it is encouraging that the CLM turns out to be useful in overcoming the severe sign problem that occurs in applying standard Monte Carlo methods to this model. On the other hand, further developments in solving the sign problem might be necessary in order to approach the target theory corresponding to $(s, k)=(0,0)$ including the fermionic matrices. We hope that such developments eventually enable us to answer profound questions concerning the origin of our Universe.

\section{Acknowledgements}

The author would like to thank K.N. Anagnostopoulos, T. Aoki, T. Azuma, M. Hirasawa, Y. Ito, H. Kawai, S.K. Papadoudis, H. Steinacker and A. Tsuchiya for valuable discussions. He is also grateful to K.N. Anagnostopoulos and G. Zoupanos for their warm hospitality during the Summer Institute. This work was supported by MEXT as "Program for Promoting Researches on the Supercomputer Fugaku" (Simulation for basic science: from fundamental laws of particles to creation of nuclei). It was also supported by computational time granted from the Greek Research \& Technology Network (GRNET) in the National HPC facility -ARIS- under project IDs IIB10D and LIKKT.

\section{References}

[1] S.-W. Kim, J. Nishimura, and A. Tsuchiya, Expanding (3+1)-dimensional universe from a Lorentzian matrix model for superstring theory in (9+1)-dimensions, Phys. Rev. Lett. 108 (2012) 011601, arXiv:1108.1540.

[2] N. Ishibashi, H. Kawai, Y. Kitazawa, and A. Tsuchiya, A Large N reduced model as superstring, Nucl. Phys. B498 (1997) 467-491, hep-th/9612115.

[3] M. Fukuma, H. Kawai, Y. Kitazawa, and A. Tsuchiya, String field theory from IIB matrix model, Nucl. Phys. B 510 (1998) 158-174, hep-th/9705128.

[4] G. Parisi, On Complex Probabilities, Phys. Lett. B131 (1983) 393-395.

[5] J. R. Klauder, Coherent State Langevin Equations for Canonical Quantum Systems With Applications to the Quantized Hall Effect, Phys. Rev. A29 (1984) 2036-2047.

[6] G. Aarts, F. A. James, E. Seiler, and I.-O. Stamatescu, Adaptive stepsize and instabilities in complex Langevin dynamics, Phys. Lett. B687 (2010) 154-159, arXiv:0912.0617.

[7] G. Aarts, E. Seiler, and I.-O. Stamatescu, The Complex Langevin method: When can it be trusted?, Phys. Rev. D81 (2010) 054508, arXiv:0912.3360.

[8] G. Aarts, F. A. James, E. Seiler, and I.-O. Stamatescu, Complex Langevin: Etiology and Diagnostics of its Main Problem, Eur. Phys. J. C71 (2011) 1756, arXiv:1101.3270.

[9] E. Seiler, D. Sexty, and I.-O. Stamatescu, Gauge cooling in complex Langevin for QCD with heavy quarks, Phys.Lett. B723 (2013) 213-216, arXiv:1211.3709.

[10] J. Nishimura and S. Shimasaki, New insights into the problem with a singular drift term in the complex Langevin method, Phys. Rev. D92 (2015), no. 1 011501, arXiv:1504.08359. 
[11] K. Nagata, J. Nishimura, and S. Shimasaki, Justification of the complex Langevin method with the gauge cooling procedure, PTEP 2016 (2016), no. 1 013B01, arXiv:1508.02377.

[12] K. Nagata, J. Nishimura, and S. Shimasaki, Argument for justification of the complex Langevin method and the condition for correct convergence, Phys. Rev. D94 (2016), no. 11114515 , arXiv:1606.07627.

[13] Y. Ito and J. Nishimura, The complex Langevin analysis of spontaneous symmetry breaking induced by complex fermion determinant, JHEP 12 (2016) 009, arXiv:1609.04501].

[14] G. Aarts, E. Seiler, D. Sexty, and I.-O. Stamatescu, Complex Langevin dynamics and zeroes of the fermion determinant, JHEP 05 (2017) 044, arXiv: 1701.02322.

[15] M. Scherzer, E. Seiler, D. Sexty, and I.-O. Stamatescu, Complex Langevin and boundary terms, Phys. Rev. D 99 (2019), no. 1 014512, arXiv:1808.05187.

[16] K. N. Anagnostopoulos, T. Azuma, Y. Ito, J. Nishimura, and S. K. Papadoudis, Complex Langevin analysis of the spontaneous symmetry breaking in dimensionally reduced super Yang-Mills models, JHEP 02 (2018) 151, arXiv: 1712.07562.

[17] T. Aoyama, J. Nishimura, and T. Okubo, Spontaneous breaking of the rotational symmetry in dimensionally reduced super Yang-Mills models, Prog. Theor. Phys. 125 (2011) 537-563, arXiv:1007.0883.

[18] K. N. Anagnostopoulos, T. Azuma, Y. Ito, J. Nishimura, T. Okubo, and S. Kovalkov Papadoudis, Complex Langevin analysis of the spontaneous breaking of 10D rotational symmetry in the Euclidean IKKT matrix model, arXiv:2002.07410,

[19] J. Nishimura, T. Okubo, and F. Sugino, Systematic study of the SO(10) symmetry breaking vacua in the matrix model for type IIB superstrings, JHEP 10 (2011) 135, arXiv:1108.1293.

[20] Y. Ito, S.-W. Kim, Y. Koizuka, J. Nishimura, and A. Tsuchiya, A renormalization group method for studying the early universe in the Lorentzian IIB matrix model, PTEP 2014 (2014), no. $8083 \mathrm{~B} 01$, arXiv:1312.5415.

[21] Y. Ito, J. Nishimura, and A. Tsuchiya, Power-law expansion of the Universe from the bosonic Lorentzian type IIB matrix model, JHEP 11 (2015) 070, arXiv:1506.04795.

[22] Y. Ito, J. Nishimura, and A. Tsuchiya, Universality and the dynamical space-time dimensionality in the Lorentzian type IIB matrix model, JHEP 03 (2017) 143, arXiv: 1701.07783.

[23] T. Azuma, Y. Ito, J. Nishimura, and A. Tsuchiya, A new method for probing the late-time dynamics in the Lorentzian type IIB matrix model, PTEP 2017 (2017), no. 8 083B03, arXiv: 1705.07812].

[24] H. S. Yang, Emergent Spacetime and Cosmic Inflation I \& II, arXiv:1503.00712

[25] K. K. Kim, S. Koh, and H. S. Yang, Expanding Universe and Dynamical Compactification Using Yang-Mills Instantons, JHEP 12 (2018) 085, arXiv:1810.12291.

[26] K. Tomita, Fluctuations of the cosmic background radiation appearing in the 10-dimensional cosmological model, PTEP 2015 (2015), no. 12 123E01, arXiv:1511.08583.

[27] T. Aoki, M. Hirasawa, Y. Ito, J. Nishimura, and A. Tsuchiya, On the structure of the emergent $3 d$ expanding space in the Lorentzian type IIB matrix model, PTEP 2019 (2019), no. 9 093B03, arXiv:1904.05914.

[28] K. Hatakeyama, A. Matsumoto, J. Nishimura, A. Tsuchiya, and A. Yosprakob, The emergence of expanding space-time and intersecting D-branes from classical solutions in the Lorentzian type IIB matrix model, PTEP 2020 (2020), no. 4 043B10, arXiv: 1911.08132. 
[29] J. Nishimura and A. Tsuchiya, Complex Langevin analysis of the space-time structure in the Lorentzian type IIB matrix model, JHEP 06 (2019) 077, arXiv:1904.05919.

[30] W. Krauth, H. Nicolai, and M. Staudacher, Monte Carlo approach to M theory, Phys. Lett. B431 (1998) 31-41, hep-th/9803117.

[31] P. Austing and J. F. Wheater, Convergent Yang-Mills matrix theories, JHEP 04 (2001) 019, hep-th/0103159.

[32] K. N. Anagnostopoulos, T. Azuma, and J. Nishimura, Monte Carlo studies of the spontaneous rotational symmetry breaking in dimensionally reduced super Yang-Mills models, JHEP 11 (2013) 009, arXiv:1306.6135.

[33] A. Mollgaard and K. Splittorff, Complex Langevin Dynamics for chiral Random Matrix Theory, Phys.Rev. D88 (2013), no. 11 116007, arXiv:1309.4335.

[34] K. Nagata, J. Nishimura, and S. Shimasaki, Testing the criterion for correct convergence in the complex Langevin method, JHEP 05 (2018) 004, arXiv:1802.01876.

[35] S.-W. Kim, J. Nishimura, and A. Tsuchiya, Expanding universe as a classical solution in the Lorentzian matrix model for nonperturbative superstring theory, Phys. Rev. D86 (2012) 027901, arXiv:1110.4803.

[36] S.-W. Kim, J. Nishimura, and A. Tsuchiya, Late time behaviors of the expanding universe in the IIB matrix model, JHEP 10 (2012) 147, arXiv:1208.0711].

[37] F. R. Klinkhamer, On the emergence of an expanding universe from a Lorentzian matrix model, arXiv:1912.12229

[38] A. Chaney, L. Lu, and A. Stern, Matrix Model Approach to Cosmology, Phys. Rev. D93 (2016), no. 6 064074, arXiv:1511.06816.

[39] A. Chaney, L. Lu, and A. Stern, Lorentzian Fuzzy Spheres, Phys. Rev. D92 (2015), no. 6064021 , arXiv:1506.03505.

[40] A. Chaney and A. Stern, Fuzzy CP2 spacetimes, Phys. Rev. D95 (2017), no. 4 046001, arXiv:1612.01964.

[41] A. Stern and C. Xu, Signature change in matrix model solutions, Phys. Rev. D98 (2018), no. 8 086015, arXiv:1808.07963.

[42] H. C. Steinacker, Cosmological space-times with resolved Big Bang in Yang-Mills matrix models, JHEP 02 (2018) 033, arXiv:1709.10480.

[43] H. C. Steinacker, Quantized open FRW cosmology from Yang-Mills matrix models, Phys. Lett. $\mathbf{B 7 8 2}$ (2018) 176-180, arXiv:1710.11495.

[44] A. Chatzistavrakidis, H. Steinacker, and G. Zoupanos, Intersecting branes and a standard model realization in matrix models, JHEP 09 (2011) 115, arXiv:1107.0265.

[45] J. Nishimura and A. Tsuchiya, Realizing chiral fermions in the type IIB matrix model at finite N, JHEP 12 (2013) 002, arXiv: 1305.5547].

[46] H. C. Steinacker and J. Zahn, An extended standard model and its Higgs geometry from the matrix model, PTEP 2014 (2014), no. 8 083B03, arXiv:1401.2020.

[47] H. Aoki, J. Nishimura, and A. Tsuchiya, Realizing three generations of the Standard Model fermions in the type IIB matrix model, JHEP 05 (2014) 131, arXiv:1401.7848. 